\title{
ETNOBOTÂNICA NO ACRE: TRÊS DÉCADAS DE PESQUISAS CIENTÍFICAS REALIZADAS NO ESTADO (1990-2020)
}

\author{
ETHNOBOTANY IN ACRE: THREE DECADES OF SCIENCE RESEARCH IN THE STATE \\ $(1990-2020)$
}

Tomaz Ribeiro LANZA ${ }^{1 *}$; Lin Chau MING2; Moacir HAVERROTH ${ }^{3}$; Almecina Balbino FERREIRA ${ }^{4}$

\begin{abstract}
1. Universidade Estadual Paulista, Faculdade de Ciências Agronômicas, Departamento de Horticultura, Botucatu, São Paulo, Brasil. tomazlanza@ gmail.com.; 2. Universidade Estadual Paulista, Faculdade de Ciências Agronômicas, Departamento de Horticultura, Botucatu, São Paulo, Brasil. linming@ @unesp.br; 3. Embrapa Acre, Rio Branco, Acre, Brasil. moacir.haverrot@embrapa.br;; 4. Universidade Federal do Acre, Centro de Ciências Biológicas e da Natureza, Rio Branco, Acre, Brasil.almecina@yahoo.com.br
\end{abstract}

\section{RESUMO}

Submitted: 13/01/2020; Accepted: 02/03/2020

O presente trabalho teve como objetivo traçar um panorama dos 30 anos de pesquisa com etnobotânica no Estado do Acre a partir de um levantamento bibliográfico sistemático e aprofundado. A metodologia utilizada foi de busca por palavras-chave, sendo utilizadas nas pesquisas "etnobotânica Acre", "ethnobotany Acre Brazil", "botânica econômica Acre", "economic botany Acre Brazil". A pesquisa foi realizada em bases bibliográficas, bibliotecas digitais e físicas, periódicos, livros e acervos acadêmicos, nacionais e internacionais. Foram encontrados 111 trabalhos publicados entre 1990 e 2019, sendo que, aproximadamente, 86 trabalhos ( $77 \%$ do total) foram publicados na última década. Foram encontrados diferentes tipos de publicações, sendo elas capítulo de livro, artigo científico, resumo em congresso, livros, dissertações de mestrado, teses de doutorado, documento técnico e tese de livre docência. As temáticas mais estudadas nesses trabalhos foram plantas medicinais, agrobiodiversidade, plantas úteis e plantas alimentícias, que, juntas, representam aproximadamente $95 \%$ dos estudos. As populações tradicionais mais estudadas foram seringueiros e indígenas. Esses estudos foram desenvolvidos em diferentes municípios do Estado do Acre, sendo os mais representados Xapurí, com 17, Feijó, com 15, e Rio Branco, com 13 estudos. Esses resultados evidenciam como as pesquisas em etnobotânica no Acre são recentes e, além disso, quais as regiões e populações ainda podem ser estudadas.

Palavras-chave: Amazônia; Revisão bibliográfica; Etnobotânica.

\section{ABSTRACT}

This paper aims to draw an overview of 30 years of research with ethnobotany in the state of Acre from a systematic and in-depth bibliographic survey. The methodology used was to search for keywords, being used in the researches "ethnobotany Acre", "ethnobotany Acre Brazil", "economic botany Acre", "economic botany Acre Brazil". The research was conducted in bibliographic bases, digital and physical libraries, journals, books and academic collections, national and international. We found 111 papers published between 1990 and 2019, and approximately 86 papers (77\% of the total) were published in the last decade. Different types of publications were found, such as book chapter, scientific article, congress abstract, books, master's dissertations, doctoral theses, technical document and free teaching thesis. The most studied themes in these works were medicinal plants, agrobiodiversity, useful plants and food plants, which together represent approximately $95 \%$ of the studies. The most studied traditional populations were rubber tappers and indigenous peoples. These studies were developed in different municipalities of the state of Acre, the most represented being Xapurí with 17, Bean with 15, and Rio Branco with 13 studies. These results show how recent research on ethnobotany in Acre State, and, in addition, which regions and populations can still be studied.

Keywords: Amazon; Literature review; Ethnobotany

\section{INTRODUÇÃO}

O Estado do Acre corresponde a menos de $2 \%$ do território brasileiro, sendo um dos Estados menos povoados do país, com cerca de $4,47 \mathrm{hab} / \mathrm{km}^{2}$. Sua capital é Rio Branco, conhecida por "Cidade Verde" ou "Capital da Natureza", com população estimada de 407 mil habitantes. Faz fronteiras com o 
Amazonas ao norte, Rondônia ao leste, a Bolívia ao sudeste e o Peru ao sul e oeste. As cidades mais populosas do Estado são Rio Branco, Cruzeiro do Sul e Sena Madureira (IBGE, 2019).

Em relação à cobertura vegetal e ao uso do solo, o Estado apresenta cerca de $90 \%$ de seu território coberto de áreas florestais (MAPBIOMAS, 2018), tendo grande parte do seu território considerado como área prioritária para conservação da biodiversidade (BRASIL, 2019). Existem, atualmente, 21 unidades de conservação (UC's), somando aproximadamente 53.483,62 $\mathrm{km}^{2}$ de áreas protegidas (BRASIL, 2019). A importância do Acre na diversidade de plantas existentes no Brasil também é relevante, uma vez que, das 12.414 espécies de plantas identificadas na região norte do Brasil, 4.028 espécies, ou 32\% deste total, ocorrem no Estado, sendo que 58 são consideradas endêmicas (ZAPPI et al., 2015).

Com relação à diversidade de populações tradicionais, só considerando as Terras Indígenas (TI), o Estado possui, atualmente, 35 territórios homologados ou em processo de homologação, onde vivem, atualmente, 15 etnias indígenas distintas (FUNAI, 2019; ISA, 2019; CPI, 2019). Além de populações indígenas, o Acre conta com uma grande diversidade de populações ribeirinhas, extrativistas e camponesas distribuídas por toda sua extensão, sendo essas populações detentoras de amplo conhecimento associados à flora local.

Ao longo das últimas décadas, diversos pesquisadores e pesquisadoras têm olhado o Estado do Acre com grande potencial para a realização de pesquisas botânicas e etnobotânicas, seja pelo alto grau de conservação dos ecossistemas naturais presentes, como também pela grande diversidade de populações tradicionais que vivem na região (DINERSTEIN et al. 1995; OLSON et al. 1996; MING, 2006; DALY e SILVEIRA, 2009; HAVERROTH, 2018).

O desenvolvimento de pesquisas com etnobotânica é estratégico na conservação socioambiental de uma região geográfica, valorizando o conhecimento tradicional das populações locais e subsidiando pesquisas e prospecções mais aprofundadas de espécies úteis com diferentes potenciais de usos. Os primeiros conceitos acerca dessa ciência definiam que a etnobotânica tem como objetivo estudar o uso e a distribuição de plantas por populações humanas e a maneira como se dá a transferência desse conhecimento botânico entre as gerações (HARSHBERGER, 1893). Esses estudos avaliam a manipulação humana de plantas, as tecnologias adaptadas utilizadas, as formas de classificação, os sistemas agrícolas tradicionais e substâncias utilizadas por essas populações (SCHULTES, 1994).

De acordo com autores mais recentes, a etnobotânica tem como objetivo estudar a inter-relação entre pessoas e as plantas em seu ambiente, porém, com o passar do tempo, essa ciência vem desenvolvendo tanto uma perspectiva teórica quanto metodológica (ALBUQUERQUE e HANAZAKI, 2009; RITTER et al., 2015). A etnobotânica é muito mais do que uma classificação de plantas. Hamilton et al. (2003) destacam que esses estudos devem registrar não apenas listas de usos de plantas, mas uma visão da própria vida da população estudada. Sendo assim, é importante destacar que, apesar de teoricamente ser uma ciência nova, na prática, é uma disciplina antiga, com um papel relevante no desenvolvimento da sociedade e em aspectos biológicos, culturais e na conservação da sociobiodiversidade (HAMILTON et al., 2003).

Considerando a relevância da região frente à sociobiodiversidade existente, o presente trabalho tem como objetivo traçar um panorama dos 30 anos de etnobotânica no Estado no Acre a partir das publicações encontradas na literatura. Além disso, pretende-se também incentivar novas pesquisas no Estado a fim de valorizar a diversidade sociocultural da região e dar visibilidade a essas populações e suas lutas territoriais.

\section{MATERIAL E MÉTODOS}

Para realização da pesquisa, foi utilizada, como critério, a busca por trabalhos científicos acadêmicos, não levando em consideração relatos na literatura nem documentos históricos, antropológicos, literários ou de outra natureza realizados no Estado do Acre. Dessa forma, considerouse, como marco inicial das pesquisas em etnobotânica no Estado, a publicação do primeiro trabalho científico sob essa temática.

A pesquisa foi realizada em bases bibliográficas digitais, periódicos, livros e acervos acadêmicos, nacionais e internacionais. As principais bases digitais utilizadas foram Web of Science, Scielo, Google Acadêmico, Periódicos Capes e Scopus (Elsevier). Além disso, foram consultados periódicos relevantes na área de etnobiologia, como Economic Botany, Acta Botânica Brasílica, Acta Amazônica, Revista Brasileira de Plantas Medicinais, Rodriguesia, Ethnoscientia, Boletim do Museu Paraense Emílio Goeldi, entre outros. 
As buscas bibliográficas foram feitas a partir de palavras-chave, como "etnobotânica Acre", "ethnobotany Acre Brazil", "botânica econômica Acre", "economic botany Acre Brazil", adicionadas nos campos de pesquisa "título", "resumo" e "palavras-chave" das bases consultadas. Após esgotamento de trabalhos em acervos acadêmicos a partir de palavras-chave, os principais autores identificados foram consultados para contribuírem no presente trabalho.

Após a identificação das pesquisas que atendiam aos critérios metodológicos estabelecidos, estes foram classificados de acordo com: (1) ano de publicação, (2) tipo de publicação, (3) município onde foi realizada, (4) população estudada, (5) enfoque da pesquisa e (6) abrangência do trabalho. Os dados foram registrados em planilhas do programa Microsoft Excel, processados e analisados quantitativa e qualitativamente.

\section{RESULTADOS E DISCUSSÃO}

As pesquisas com etnobotânica no Brasil são recentes, com poucos trabalhos realizados no bioma amazônico quando comparados a outros biomas, como Mata Atlântica, Cerrado e Caatinga (RITTER et al., 2015). As primeiras publicações em etnobotânica no Brasil com abordagens mais recentes são representadas por Emmerich e Senna (1980), Balée et al. (1985), Prance (1987), Amorozo e Gely (1988), entre outros. No Acre não é diferente, evidenciando um potencial investigativo do Estado ainda pouco explorado pela ciência.

Diversos autores vêm se empenhando, nas últimas décadas, para compreender melhor esse Estado tão pouco conhecido e as populações que ali vivem. A complexidade geográfica da região, a logística restrita de deslocamento no Estado e a dificuldade de acesso nos períodos de inverno amazônico dificultam o desenvolvimento de pesquisas de campo em regiões mais isoladas, refletindo em baixo número de trabalhos científicos quando comparado a outros Estados do bioma amazônico.

De acordo com levantamento realizado, até o momento da publicação desse trabalho foram publicados 111 trabalhos científicos na área de etnobotânica no Estado do Acre entre 1990 e 2020, não sendo encontrado nenhum trabalho realizado antes a esse período (Figura 1). A maior parte dos trabalhos foi publicada a partir do ano de 2010, sendo que, do total de trabalhos encontrados, $77 \%$ das publicações ocorreram entre 2010 e 2019, demonstrando uma intensificação em compreender a relação de populações tradicionais com plantas no Estado durante esse período.

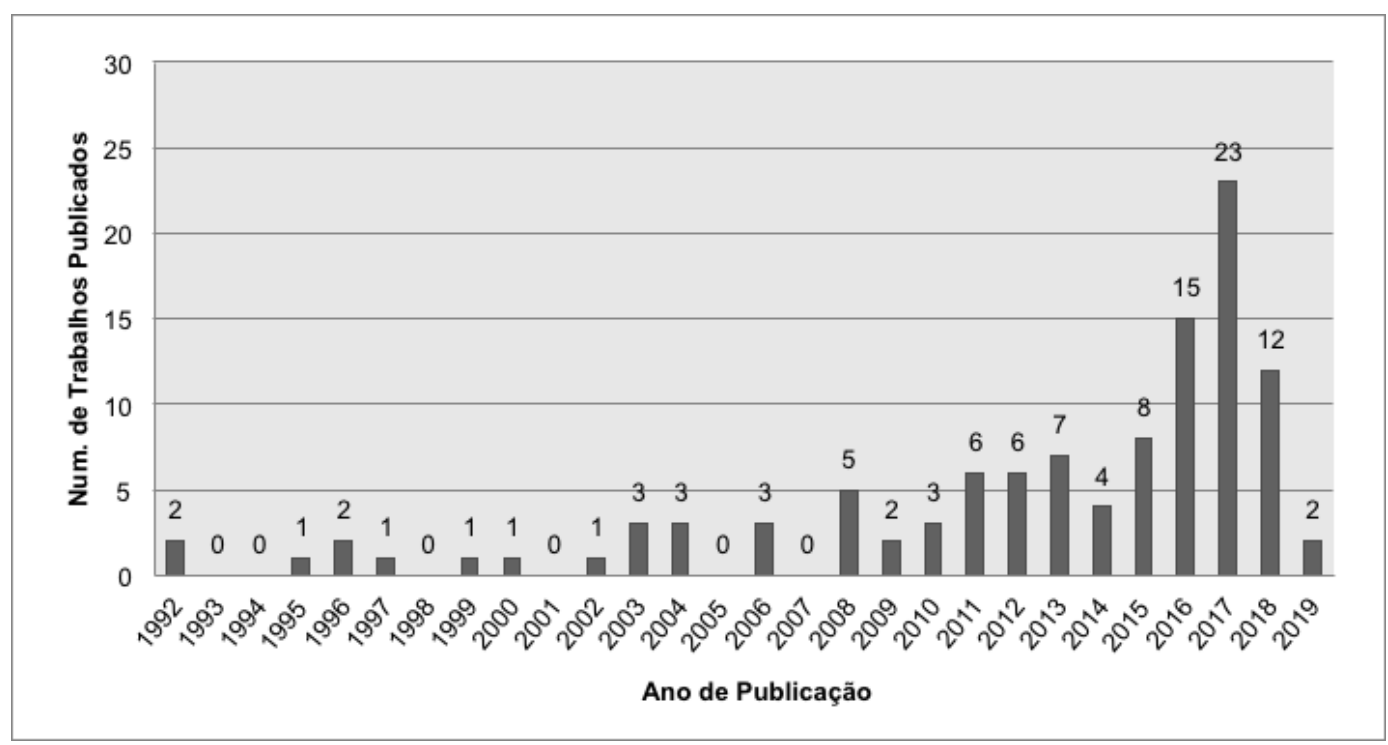

Figura 1: Número de publicações de etnobotânica no Estado do Acre por ano.

Até o ano de 2010, somente 25 trabalhos haviam sido publicados na área, ou $23 \%$ do total. Observa-se, na Figura 1, que entre os anos de 1992 e 2008 não mais que três trabalhos com etnobotânica foram publicados por ano. Isso demonstra que os estudos etnobotânicos realizados são recentes e que, até o respectivo ano, pouco se sabia, cientificamente, a respeito do uso de plantas por comunidades tradicionais da região.

Os anos com maior número de publicações foram 2016 (15 publicações), 2017 (23 publicações) e 2018 (12 publicações), decorrentes, principalmente, de projetos realizados por pesquisadores da 
Embrapa Acre e outros parceiros, como da Universidade Estadual Paulista (UNESP) e Universidade Federal do Acre (UFAC). Nesse período, ampliaram-se os esforços em estudar as populações tradicionais em regiões ainda pouco estudadas, como os municípios de Feijó e Cruzeiro do Sul, principalmente por conta da melhoria nos acessos a essas regiões.

Com relação à localização desses trabalhos, foi possível identificar que 11 municípios (50\%), do total de 22 municípios do Estado, já tiveram alguma pesquisa de etnobotânica realizada (Figura 2). Esses trabalhos foram desenvolvidos em diferentes municípios, sendo os mais representativos Feijó (27 publicações), Xapuri (26), Cruzeiro do Sul (19) e Rio Branco (16); soma desses representa 80\% dos trabalhos publicados.

Esses resultados sugerem que existe um grande potencial para realização de novas pesquisas em etnobotânica nos municípios ainda não estudados, como é o caso de Porto Walter, Santa Rosa do Purus, Manoel Urbano, Santa Rosa do Purus, Assis Brasil, Brasiléia, Epitaciolândia, Plácido de Castro, Senador Guiomard, Acrelândia e Porto Acre.

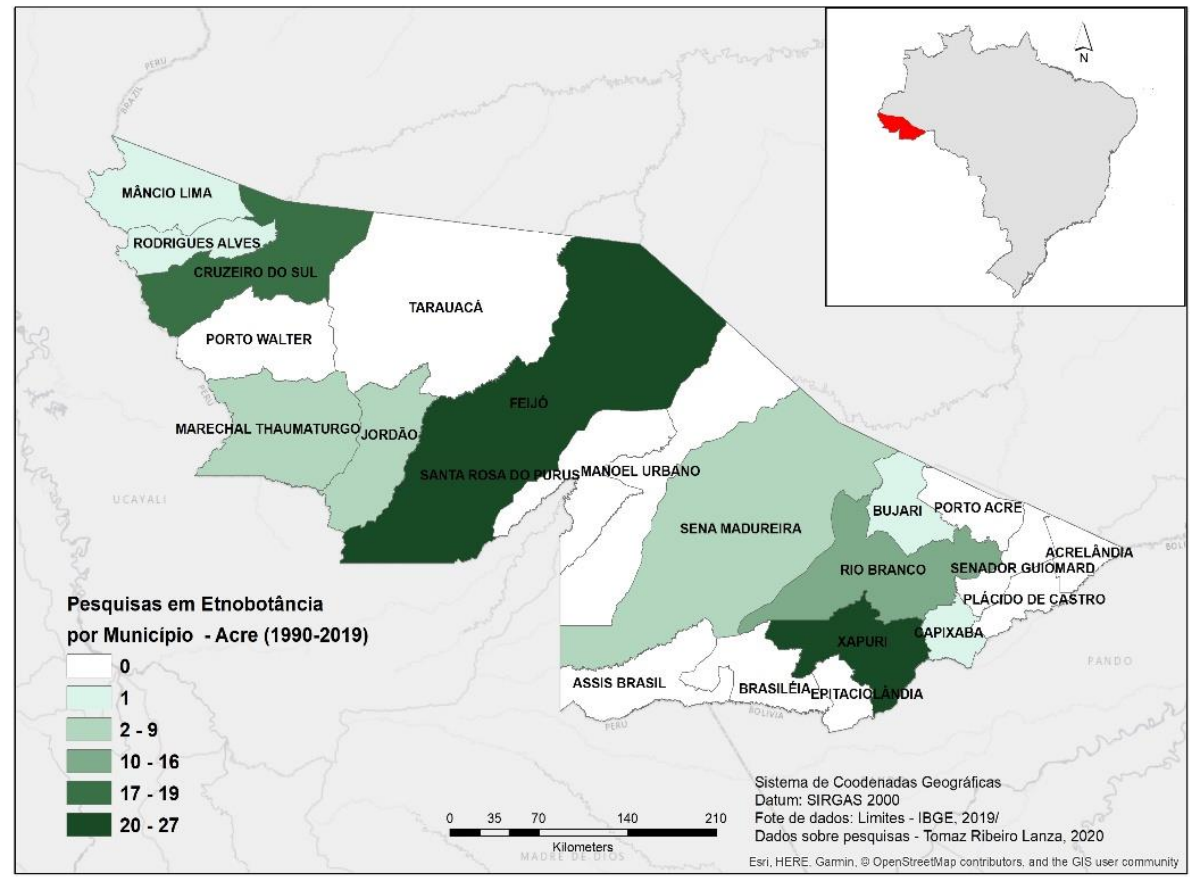

Figura 2: Pesquisas em etnobotânica por município do Estado do Acre.

Quanto ao formato das publicações, as mesmas estão divididas em oito formatos principais, sendo elas: Capítulo de Livro, Resumo de Congresso, Artigo Científico, Dissertação de Mestrado, Livro, Tese de Doutorado, Documento Técnico e Tese de Livre Docência. Ao analisar os dados, foi identificado que há predominantemente trabalhos no formato de capítulo de livro, anais de congresso e artigos científicos, que, juntas, somam $76 \%$ das publicações encontradas. Também foram encontrados oito livros publicados, dois documentos técnicos e um trabalho de livre docência (Figura 3).

Com relação aos livros, cabe destacar trabalhos importantes que envolveram populações tradicionais do Acre, como o amplo estudo de conhecimento tradicional de plantas realizado por Da Cunha e Almeida (2012) e o estudo de palmeiras úteis da Amazônia publicado por Smith (2014), onde ambos levaram em consideração o conhecimento de populações tradicionais do Estado.

No livro intitulado Enciclopédia da floresta, escrito por Da Cunha e Almeida (2012), os autores buscaram olhar a região através de diferentes pontos de vista: sua diversificada biota, sua história recente e das pessoas que a habitam, com uma compreensão própria dessas pessoas sobre o meio em que vivem. O livro é muito completo e registra tanto o esforço intelectual dos cientistas, como dos detentores de um conhecimento, tradicional, com respeito a uma única floresta e duas epistemologias, por meio das quais o leitor aprende sobre a mesma floresta. 


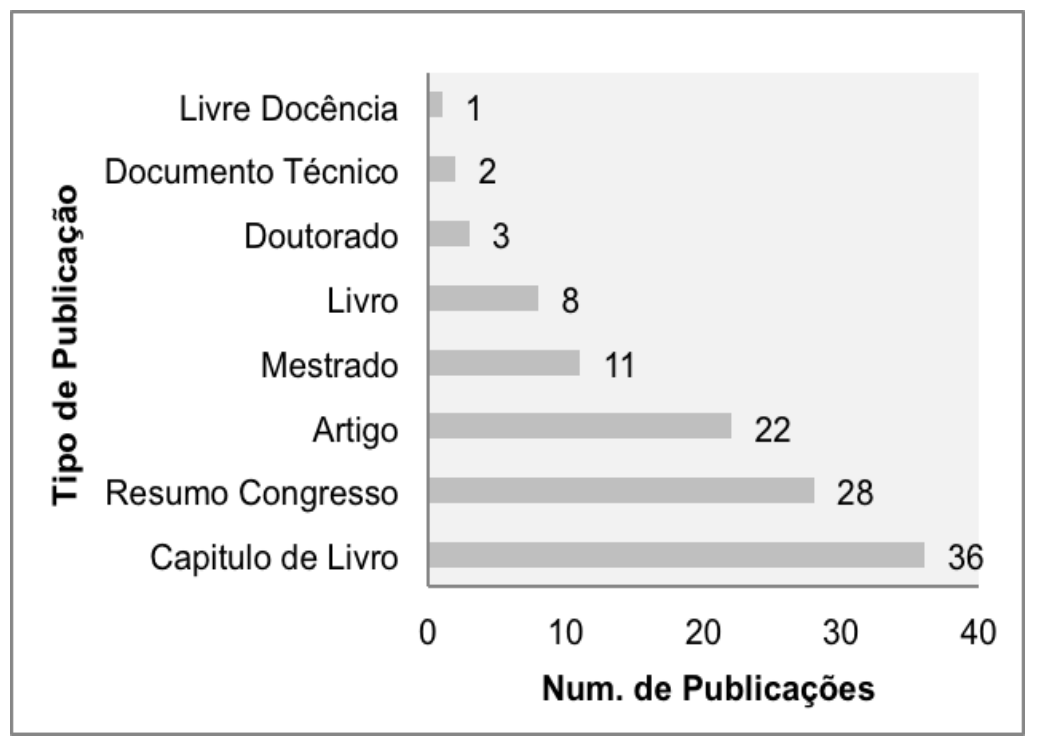

Figura 3: Número de publicações por tipo de publicação dos estudos de etnobotânica no Acre.

Já o livro Palms and people in the Amazon, Smith (2014) investigou o grau em que as paisagens do Estado do Acre e de outras regiões amazônicas foram enriquecidas com palmeiras por atividades humanas e a importância que essas plantas possuem para as populações locais até os dias atuais.

Com relação aos trabalhos de conclusão de graduação e pós-graduação (mestrado e doutorado), foram encontradas 11 dissertações de mestrado, três teses de doutorado e nenhuma monografia de graduação, representando, juntas, somente $12 \%$ do total de publicações totais identificadas. Sugere-se que esse baixo índice de trabalhos realizados para conclusões de graduação e/ou pós-graduação são decorrentes, principalmente, das dificuldades relativas à realização de pesquisas na região, na maioria dos casos, com altos custos de investimentos e logísticas complexas de acesso ao interior dos municípios.

Esses resultados sugerem que há uma demanda existente no Estado para esse tipo de trabalho mais amplo relacionado a trabalhos finais de graduações e pesquisas de pós-graduação, sendo uma grande oportunidade para universidades do Acre e de todo o Brasil realizarem novas pesquisas sob essa temática.

Quando avaliamos as populações tradicionais estudadas, percebe-se que as populações mais pesquisadas foram as de seringueiros e indígenas, somando, juntas, $57 \%$ das publicações encontradas. $\mathrm{Na}$ década de 1990, as pesquisas concentraram esforços em estudar as populações extrativistas do Estado, em especial de seringueiros.

Após a morte de Chico Mendes, a visibilidade nacional e internacional da região cresceu significativamente, decorrente do momento histórico que vivia, de conflitos ambientais e políticos recorrentes. Dessa forma, ampliaram-se os esforços em dimensionar o conhecimento acerca de plantas úteis utilizadas por essas populações, valorizando e ampliando ainda mais a visibilidade e os direitos dessas comunidades.

Como pode ser observado na Figura 4, além de seringueiros e indígenas, outras populações foram estudadas nessas pesquisas, sendo elas populações amazônicas no geral, urbanas, agricultores familiares, ribeirinhos e raizeiros.

Os primeiros trabalhos de etnobotânica realizados no Estado do Acre foram publicados por Emperaire e Delavaux (1992) e Kainer e Duryea (1992), que pesquisaram a relação dessas populações com as plantas úteis de áreas de Reservas Extrativistas (RESEX), dos municípios de Marechal Thaumaturgo e Xapuri. Após esse período, outros autores também se interessaram por essas populações, como Ming (1995), que realizou a primeira tese de doutorado com etnobotânica, cujo enfoque foram as plantas medicinais utilizadas pelos seringueiros na RESEX Chico Mendes na parte localizada no município de Xapurí. Durante sua pesquisa, Ming (1995) pôde conviver som os seringueiros e compreender o amplo conhecimento acumulado sobre plantas medicinais amazônicas, sendo um trabalho importante para dar visibilidade a essas populações tradicionais até então pouco conhecidas. 


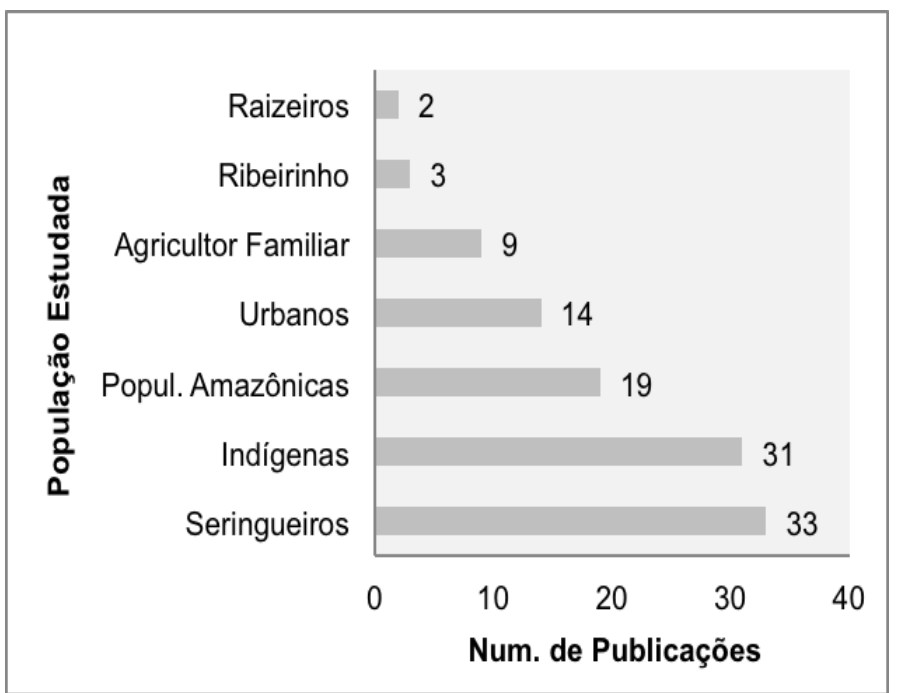

Figura 4: Número de publicações por população dos estudos de etnobotânica no Acre.

O interesse pelas populações indígenas, muito numerosas e diversificadas em todo o Estado, iniciaram-se com o trabalho pioneiro de Ehringhaus (1996), cujo enfoque foram as plantas medicinais do gênero Piper ssp., da família botânica Piperaceae, utilizadas pela etnia Kaxinawá em Terra Indígena do município de Jordão. Essa etnia, além de ser a mais populosa do Acre (ISA, 2019), foi a mais pesquisada, concentrando $80 \%$ das publicações de etnobotânica envolvendo conhecimento indígena na região. Esses dados mostram que, apesar de grande diversidade de etnias no Acre, pouco se sabe a respeito das demais populações indígenas que vivem ali.

Os demais trabalhos nessa linha foram realizados com outras etnias indígenas, como, por exemplo, Lenaerts (2010), com estudo etnográfico e antropológico das plantas medicinais utilizadas pela etnia Ashaninka no Acre e países vizinhos; Haverroth et al. (2010), em pesquisas realizadas com a etnia Kulina, onde identificaram aproximadamente 193 espécies de plantas medicinais sendo utilizadas por essa população; e Costa (2019), em levantamento de plantas alimentícias realizado com a etnia Shanenawa.

Cabe destacar que, das 15 etnias que vivem no Estado, 11 não tiveram seus conhecimentos sobre plantas úteis publicados até o presente momento, sendo elas: Apolima-Arara, Shawãdawa ou Arara, Katukina ou Noke Koi, Manchineri, Puyanawa, Yawanawá, Nukini, Kuntanawa, Nawa, Yaminawa ou Jaminawa e Isolados do Alto Envira (sem etnia definida). Esses dados reforçam a importância de se ampliarem os estudos com populações indígenas de outras etnias no Estado.

Diversos trabalhos, 19 do total, incorporaram diferentes populações tradicionais amazônicas em um mesmo trabalho, reunindo informações diversas sobre uso de plantas, como é o caso de Katz (2010), Ferreira et al. (2015) e Siviero et al. (2016).

Com relação às temáticas mais estudadas, foi identificado que a maior parte das publicações (97\%) teve como objetivo identificar plantas medicinais (31 publicações), agrobiodiversidade (31), plantas úteis no geral (22) e plantas alimentícias (24) das comunidades estudadas (Figura 5).

Com enfoque em plantas medicinais, cabe destacar os livros desenvolvidos por Ming (2006), relativos a pesquisas realizadas na RESEX Chico Mendes, e por Îka Muru e Quinet (2011), desenvolvido com os Kaxinawá do Jordão, sendo que ambos os trabalhos tiveram como primeiro autor informantes da própria comunidade tradicional estudada. Outro trabalho importante realizado foi a dissertação de mestrado escrita por Lopes (2017), com levantamento de plantas medicinais da TI Kaxinawá de Nova Olinda, no alto Rio Envira, município de Feijó-AC, onde foram encontradas 369 plantas medicinais utilizadas por essa população.

Na temática de agrobiodiversidade, destacam-se os trabalhos realizados por Emperaire et al. (2016a), com seringueiros do município de Cruzeiro do Sul, e por Matar et al. (2017), com levantamento de variedades de feijão crioulo do vale do Rio Juruá. O foco desses e de outros trabalhos foram, principalmente, identificar as espécies cultivadas e domesticadas utilizadas por essas comunidades tradicionais. 


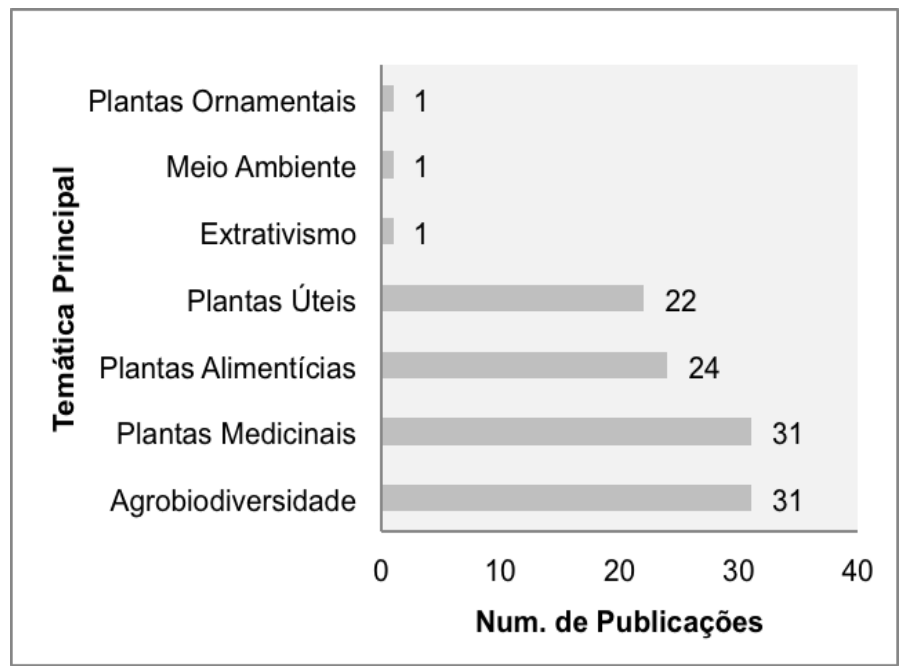

Figura 5: Número de publicações por temática principal dos estudos de etnobotânica no Acre.

Outras temáticas estudadas foram plantas úteis, como é o caso do trabalho publicado por Wallace (2004), Ming et al. (2018), e alimentícias, como é o caso de Rizzi (2011) e Van Velthem (2012), que tiveram como enfoque compreender os processos e conhecimentos envolvidos no processamento de mandioca por populações tradicionais de diferentes regiões do Estado; Lanza et al. (2018) com estudo de plantas alimentícias cultivadas e não cultivadas da população da TI Kaxinawá de Nova Olinda em Feijó-AC; e Pilnik (2019), que, além de identificar as plantas alimentícias utilizadas, buscou compreender, em seu mestrado, a alimentação e procedimentos culinários da população Kaxinawá do Alto Jordão.

Além dessas temáticas principais, foram encontrados trabalhos que tiveram como foco principal identificar plantas ornamentais (SIVIERO et al., 2014), plantas obtidas através de extrativismo específico (EMPERAIRE, 2016b) realizado com seringueira (Hevea brasiliensis (Willd. ex A.Juss.) Müll.Arg.] e trabalho cujo enfoque foi compreender a relação do conhecimento de plantas com o meio ambiente, como realizado por Santos (2008).

\section{CONCLUSÕES}

A revisão bibliográfica realizada buscou compreender a história da etnobotânica no Estado do Acre a partir dos trabalhos científicos publicados nas últimas décadas. Apesar de ser um Estado relativamente isolado dos demais, percebe-se grande esforço de instituições públicas de ensino e pesquisa para compreender a relação existente entre as populações tradicionais e as plantas utilizadas pelas mesmas.

Foram encontrados 111 trabalhos científicos de etnobotânica realizados no Acre, sendo que a maior parte desses, $77 \%$, foi publicada nos últimos dez anos. Não foi encontrada nenhuma publicação em etnobotânica realizada antes de 1992.

Os estudos com etnobotânica no Estado do Acre são recentes, sendo todos realizados somente nos últimos 30 anos. Isso mostra que ainda existem muitos locais e populações humanas a serem estudadas, abrindo oportunidades para realização de novas pesquisas.

Outro ponto importante é em relação à riqueza de populações tradicionais estudadas, que vão desde seringueiros, indígenas, agricultores familiares a ribeirinhos, raizeiros e populações urbanas. Apesar disso, observou-se que a maior parte dos trabalhos foi realizada em regiões específicas do Estado.

Com relação aos povos indígenas, observou-se que somente quatro das 15 etnias já tiveram algum tipo de levantamento etnobotânico. Isso sugere que exista uma demanda crescente em se trabalhar com as demais etnias do Estado.

\section{AGRADECIMENTOS}

À Coordenação de Aperfeiçoamento de Pessoal de Nível Superior (CAPES) pelo financiamento da pesquisa; à UNESP, EMBRAPA e UFAC pelo apoio institucional. 


\section{REFERÊNCIAS}

ALBUQUERQUE, U. P.; HANAZAKI, N. Five problems in current ethnobotanical research and some suggestions for strengthening them. Human Ecology, v. 37, n. 5, p. 653-661, 2009. Disponível em: <https://link.springer.com/article/10.1007/s10745-009-9259-9>. Acesso em: 10 dez. 2019.

AMOROZO, M. C. M.; GÉLY, A. L. Uso de plantas medicinais por caboclos do baixo Amazonas, Barcarena, PA, Brasil. Boletim do Museu Paraense Emílio Goeldi, Série Botânica v. 4, n. 1, p. 47-131, 1988.

BALÉE, W. Análise preliminar de inventário florestal e a etnobotánica Ka'apor (Maranhão). Boletim do Museu Paraense Emilio Goeldi, v. 2, n. 2, p. 141-167, 1986.

BRASIL. Áreas Prioritárias para Conservação, Uso Sustentável e Repartição de Benefícios da Biodiversidade Brasileira: Atualização - Portaria MMA nº, de 23 de janeiro de 2019. Brasília: MMA, 2019. 327p. Disponível em: <https://www.mma.gov.br/estruturas/chm/_arquivos/biodiversidade31.pdf>. Acesso em: 22 nov. 2019.

BRASIL. Cadastro Nacional de Unidades de Conservação (CNUC) - Ministério do Meio Ambiente. Brasília, 2019. Disponível em: <https://www.mma.gov.br/areas-protegidas/cadastro-nacional-de-ucs〉. Acesso em: 13 set. 2019.

COSTA, N. G. Etnobotânica de plantas alimentícias utilizadas pelo povo Shanenawa do município de Feijó, Acre. 2019. Dissertação (Mestrado em Agronomia - Horticultura). Universidade Estadual Paulista. Botucatu.

CPI. Comissão Pró Índio: Povos e Terras Indígenas do Acre. 2019. Disponível em: <http://cpiacre.org.br/conteudo/povos-e-terras-indigenas/>. Acesso em: 02 jul. 2019. 2002.

DA CUNHA, M. C.; DE ALMEIDA, M. B. Enciclopédia da floresta. São Paulo: Companhia das Letras,

DALY, D.C.; SILVEIRA, M. Primeiro catálogo da flora do Acre, Brasil. Rio Branco: EDIUFAC, 2009.

DINERSTEIN, E.; OLSON, D. M.; GRAHAM, D. J.; WEBSTER, A. L.; PRIMM, S. A., BOOKBINDER, M. P.; LEDEC, G. A conservation assessment of the terrestrial ecoregions of Latin America and the Caribbean. WWF/The World Bank, Washington, D. C., 1995.

DOS SANTOS, I. F. Caracterização socioambiental de moradores da zona ripária da bacia hidrográfica do riozinho do rôla, Rio Branco, Acre. 2008. 80 f. Dissertação (Mestrado em Ciências Florestais) - Universidade Federal de Viçosa, Viçosa.

EHRINGHAUS, C. Piper Spp.(Piperaceae) Use in an Indigenous Kaxinawá Community in Acre, Brazil: Ethnobotanical, Ecological, Phytochemical and Biological Activity Aspects. a Story of NIXPU. 1996.

EMMERICH, M.; SENNA, L. M. de. Estudos de etnobotânica no Parque Indígena do Xingu: 1. Uma periandra (Leguminosae) nova. Boletim do Museu Nacional do Rio de Janeiro Botânica, v. 57, 1980.

EMPERAIRE, L.; DELAVAUX, J.J. Etnobotância - Reserva extrativista do Alto Juruá, Acre. Relatório de Campo, Manaus: Instituto Nacional de Pesquisa da Amazônia, 1992. 77p.

FERREIRA, A.; MING, L. C.; HAVERROTH, M.; DALY, D.; CABALlERO, J.; BALLESTÉ, A. Plants Used to Treat Malaria in the Regions of Rio Branco-Acre State and Southern Amazonas State-Brazil. International Journal of Phytocosmetics and Natural Ingredients, v. 2015, p. 2-9, 2015. Disponível em: < https://www.researchgate.net/profile/Andrea_Martinez-Balleste2/publication/282413393_

Plants_Used_to_Treat_Malaria_in_the_Regions_of_Rio_Branco-Acre_State_and_Southern_Amazonas_State__Brazil/links/5717f8fe08ae30c3f9f176a2/Plants-Used-to-Treat-Malaria-in-the-Regions-of-Rio-Branco-Acre-

State-and-Southern-Amazonas-State-Brazil.pdf>. Acesso em: 10 ago. 2019.

FUNAI. Terras indígenas do Brasil. Ministério da Justiça e Segurança Pública. 2019. Disponível em: http://www.funai.gov.br/index.php/indios-no-brasil/terras-indigenas. Acesso: 06 nov. 2019.

HAMILTON, A; PEI, S. J. K.; KHAN, A. A.; LAGOS-WITTE, S.; SHINWARI, Z. K.. Os propósitos e ensinamentos da etnobotânica aplicada. Organização das Nações Unidas para a Educação, Ciência e Cultura (UNESCO), 2003.

HARSHBERGER J.W. The purposes of ethnobotany. Bot. Gaz., v. 21, n. 3, p. 146-154. 1896

HAVERROTH, M. Ensino e pesquisa em etnoecologia e etnobiologia na Região Norte do Brasil. Embrapa Acre-Artigo em periódico indexado (ALICE), 2018. Disponível em: < https://www.alice.cnptia.embrapa.br/handle/doc/1093591>. Acesso em: 13 set. 2019.

HAVERROTH, M.; NEGREIROS, P. R. M.; BARROS, L. C. P. Ethnobiology and health among the Kulina People from the Upper Envira River, State of Acre, Brazil. The Open Complementary Medicine Journal, n. 2, p. 42-57, 2010. Disponível em: < https://www.alice.cnptia.embrapa.br/handle/doc/856186>. Acesso em: 10 ago. 2019.

ÎKA MURU, A. M. M.; QUINET, A. Una Isi Kayawa: livro da cura do povo Huni Kuin do rio Jordão. Rio de Janeiro: CNC/Flora/Jardim Botânico do Rio de Janeiro e Dantes Editora, 2011.

INSTITUTO BRASILEIRO DE GEOGRAFIA E ESTATÍSTICA (IBGE). Cidades. Disponível em: <https://cidades.ibge.gov.br/brasil/brasil/ac>. Acesso em: 02 fev. 2019.

INSTITUTO SOCIOAMBIENTAL (ISA). Povos indígenas no Brasil. Brasília: ISA; 2010. Disponível em: < https://pib.socioambiental.org/pt/P\%C3\%A1gina_principal>. Acesso: 27 jan. 2019. 
KAINER, A.K.; DURYEA, M.L. Tapping women's knowledge: plant resource use in extractive reserves, Acre, Brazil. Economic Botany, v.46, n.4, p. 408-25, 1992.

KATZ, E. Cruzeiro do Sul market (Acre, Brazilian Amazon): reflection of the regional culture and agricultural diversity. In: POCHETTINO, M. L.; LADIO, A. H.; ARENAS, P. M. (Orgs.). Tradiciones y transformaciones en Etnobotánica/Traditions and transformations in Ethnobotany. San Salvador de Jujuy: Ed. CYTED, 2010. p. 525-533.

LANZA, T.R.; MING, L. C.; HAVERROTH, M.; FERREIRA, A. B. Etnobotânica de plantas alimentícias silvestres da Terra Indígena Kaxinawá de Nova Olinda, Acre, Brasil. In: CONGRESO LATINOAMERICANO AGRoECOlogíA, 7, 2018, Guayaquil. Memórias VII Congreso Latinoamericano de Agroecología. Guayaquil: SOCLA, 2018. p. 1184-1189.

LENAERTS, M. Substances, relationships and the omnipresence of the body: an overview of Ashéninka ethnomedicine (Western Amazonia). Journal of Ethnobiology and Ethnomedicine, v. 2, n. 1, p. 49, 2006. Disponível em: < https://ethnobiomed.biomedcentral.com/articles/10.1186/1746-4269-2-49>. Acesso em: 02 fev. 2019.

LOPES, B.P.C.S. Estudo etnobotânico de plantas medicinais da Terra Indígena Kaxinawá de Nova Olinda, município de Feijó, Acre. 2017. 235 f. Dissertação (Mestrado em Agronomia: Horticultura) - Faculdade de Ciências Agronômicas, Universidade Estadual Paulista, Botucatu.

MATTAR, E. P. L.; OLIVEIRA, E. D.; DE JESUS, J. C. D. S.; DE ARAÚJO, M. L.; JÚNIOR, H. C. D. S. Creole beans production systems of Jurua valley, amazon, Brasil. Indian Journal of Traditional Knowledge, v. 54, p. 619-624, 2016. Disponível em: < http://nopr.niscair.res.in/handle/123456789/35241>. Acesso em: 10 fev. 2019.

MING, L. Plantas medicinais utilizadas pelos seringueiros na Reserva Extrativista "Chico Mendes", Acre, Brasil. 1995. 180p. Tese (Doutorado em Botânica) - Instituto de Biociências, Universidade Estadual Paulista, Botucatu.

MING, L.C. Plantas medicinais na Reserva Extrativista Chico Mendes: uma visão etnobotânica. Editora UNESP, São Paulo, SP. 78p. 2006. Disponível em: < https://books.google.com.br/books?hl=ptBR\&lr=\&id=IHikHBY3Y60C\&oi=fnd\&pg=PA13\&dq=Experi\%C3\%AAncias+etnobot\%C3\%A2nicas+na+Res erva+Extrativista+Chico+Mendes\&ots=syh-4fy5w2\&sig=QU0W3rtBhAe5gchk2gZ6kAM7Cj4>. Acesso em: 23 abr. 2018.

MING, L.C.; AMOROZO, M. C. de M.; FERREIRA, A. B. Experiências etnobotânicas na Reserva Extrativista Chico Mendes. 148 p. Botucatu : Fundação de Estudos e Pesquisas Agrícolas e Florestais, 2018.

OLSON, D.; DINERSTEIN, E.; CASTRO, G.; MARAVI, E. Identifying gaps in botanical information for biodiversity conservation in Latin America and the Caribbean. World Wildlife Fund, Washington, D.C., 1996.

PRANCE, G.T. Etnobotânica de algumas tribos amazônicas. Suma Etnológica Brasileira, v. 1, p. 119-133, 1987.

MAPBIOMAS. "Projeto MapBiomas. 2018. (Coleção MapBiomas, 3; Série anual de mapas de cobertura e uso de solo do Brasil). Disponível em: 〈http://mapbiomas.org>. Acesso em: 20 ago. 2018.

RITTER, M. R.; SILVA, T. C. D.; ARAÚJO, E. D. L.; ALBUQUERQUE, U. P. Bibliometric analysis of ethnobotanical research in Brazil (1988-2013). Acta Botanica Brasilica, v. 29, n. 1, p. 113-119, 2015. Disponível em: <http://www.scielo.br/scielo.php?pid=S0102-33062015000100113\&script=sci_arttext\&tlng=es >. Acesso em: 20 set. 2018.

RIZZI R. Mandioca: processos biológicos e socioculturais associados no Alto Juruá, Acre. 2011. 169 f. Dissertação (Mestrado em Antropologia). Unicamp. Campinas

SCHULTES, R. E. The importance of ethnobotany in environmental conservation. The American Journal of Economics and Sociology, v. 53, n. 2, p. 202-206, 1994.

SIVIERO, A.; DELUNARDO, T. A.; HAVERROTH, M.; DE OLIVEIRA, L. C.; ROMAN, A. L. C.; DA SILVA MENDONÇA, Â. M.. Plantas ornamentais em quintais urbanos de Rio Branco, Brasil. Boletim do Museu Paraense Emílio Goeldi. Ciências Humanas, v. 9, n. 3, p. 797-813. 2014. Disponível em: < https://www.redalyc.org/pdf/3940/394051398015.pdf>. Acesso em: 20 set. 2018.

SIVIERO, A.; MING, L. C.; SILVEIRA, M.; DALY, D.; WALLACE, R. Etnobotânica e botânica econômica do Acre. Rio Branco: Edufac, 2016. 410 p

SMITH, N. Palms and People in the Amazon. Cham: Springer International Publishing, 500 pp. 2015.

VAN VELTHEM, L. H.; KATZ, E. A 'farinha especial': fabricação e percepção de um produto da agricultura familiar no vale do rio Juruá, Acre. Boletim do Museu Paraense Emílio Goeldi. Ciências Humanas, v. 7, n. 2, p. 435-456. 2012.

WALLACE, R. H. The effects of wealth and markets on rubber tapper use and knowledge of forest resources in Acre, Brazil. University of Florida. 2004

ZAPPI, D. C.; FILARDI, F. L. R.; LEITMAN, P.; SOUZA, V. C.; WALTER, B. M.; PIRANI, J. R.; FORZZA, R. C.. Growing knowledge: an overview of seed plant diversity in Brazil. Rodriguésia, v. 66, n. 4, p. 1085-1113, 2015. Disponível em: <http://www.scielo.br/scielo.php?pid=S2175-78602015000401085\&script=sci_arttext> Acesso em: 20 set. 2018. 